\title{
Correction to: A 14-bp stretch plays a critical role in regulating gene expression from $\beta_{1}$-tubulin promoters of basidiomycetes
}

\author{
Dong Xuan Nguyen ${ }^{1,2} \cdot$ Taku Sakaguchi $^{1} \cdot$ Takehito Nakazawa $^{1} \cdot$ Masahiro Sakamoto ${ }^{1} \cdot$ Yoichi Honda $^{1}$ (D)
}

Published online: 25 July 2019

○) Springer-Verlag GmbH Germany, part of Springer Nature 2019

\section{Correction to: Current Genetics \\ https://doi.org/10.1007/s00294-019-01014-5}

The original publication of this paper unfortunately contained three errors in Figs. 2B and 3. In Fig. 2B, the TSS site must be counted as " +1 " instead of " -1 ". And we indicated wrong sequences in Fig. 3: the construct "Control" has a missing one "A" in the BCE sequence, and the reverse direction of BCE sequence in the construct "BCEr" must be "GCGGAGTTTCAATT", not "CGCCTCAAGTTAA". For the reasons stated herein, the authors wish to notify the readers that Figs. 2B and 3 must be interpreted as the followings:

Publisher's Note Springer Nature remains neutral with regard to jurisdictional claims in published maps and institutional affiliations.

The original article can be found online at https://doi.org/10.1007/ s00294-019-01014-5.

Yoichi Honda

honda@kais.kyoto-u.ac.jp

1 Graduate School of Agriculture, Kyoto University,

Sakyo-ku, Kyoto 606-8502, Japan

2 Biotechnology Center of Ho Chi Minh City,

Ho Chi Minh City, Vietnam 


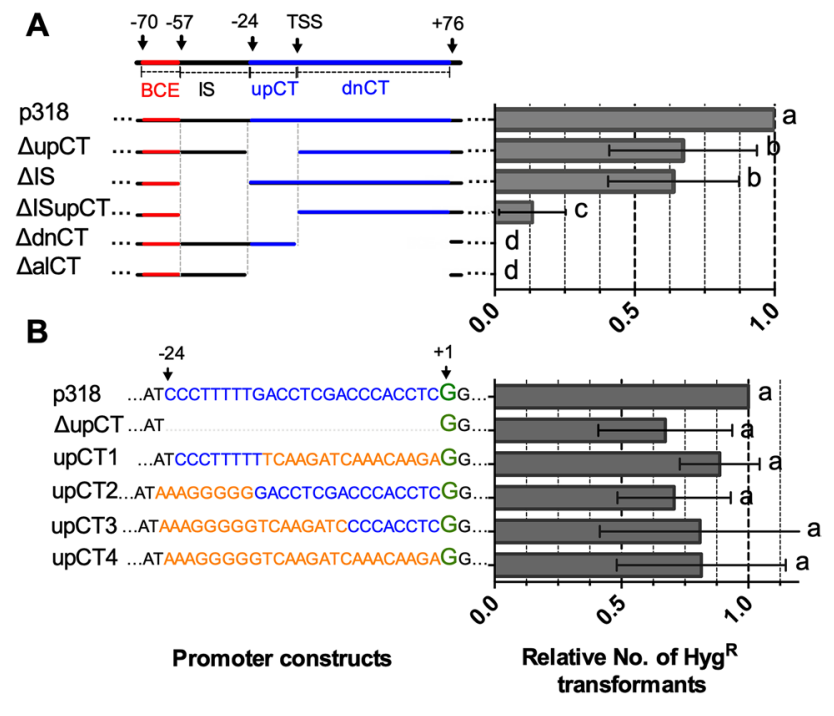

Fig. 2 Function of the CT-rich stretch and the junction sequence between BCE and TSS in the $C$. subvermispora $\beta_{l}$-tubulin promoter. Graphic depiction of the deletion analysis of the CT-rich stretch and interspace sequence between the BCE and the CT-rich stretch (A), the nucleotide substitution of upstream CT-rich stretch $(\mathbf{B})$. The relative number of Hyg-resistant transformants using each mutant variant is indicated. The red and blue lines in A represent the BCE and the CTrich stretch, respectively. Blue and orange characters in $\mathbf{A}$ represent will-type and mutant sequences, respectively. Data present in $\mathbf{A}, \mathbf{B}$ are the mean \pm SD from three independent experiments and different letters $(\mathrm{a}-\mathrm{d})$ at the right side of the columns indicate the significant differences at $p<0.05$ level between constructs

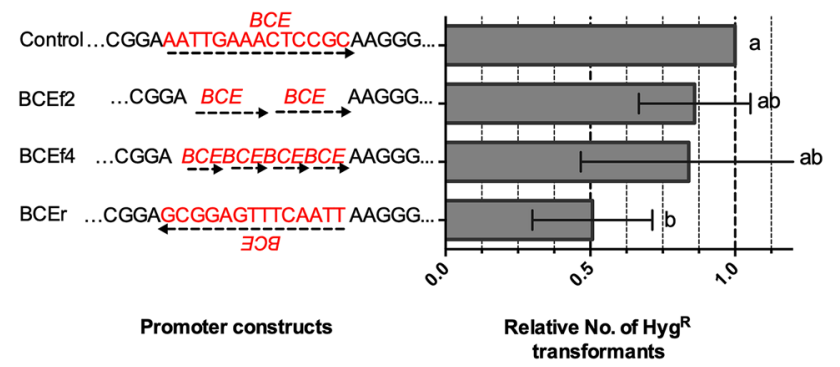

Fig. 3 Multiple insertions and reverse orientation of the BCE and the relative number of Hyg-resistant transformants. Data are the mean \pm SD from three independent experiments and different letters at the right side of the columns indicate the significant differences at $p<0.05$ level between constructs 